\title{
Exploiting the passenger ACO1-deficiency arising from 9p21 deletions to kill T-cell lymphoblastic neoplasia cells
}

\author{
Laura Gonzalez-Sanchez ${ }^{1,2,3}$, Maria A.Cobos-Fernandez ${ }^{1,2}$, Pilar Lopez-Nieva ${ }^{1,2,3, \oplus \text {, }}$ \\ Maria Villa-Morales ${ }^{1,2,3, \oplus}$, Konstantinos Stamatakis ${ }^{1}$, Jose M.Cuezva ${ }^{1,3,4, \bullet}$, \\ Jose L.Marin-Rubio ${ }^{1, \oplus}$, Irene Vazquez-Dominguez ${ }^{1,0}$, Iria Gonzalez-Vasconcellos ${ }^{1, \odot}$, \\ Eduardo Salido ${ }^{3,5, \infty}$, Pilar Llamas ${ }^{2}$, Jose L.Lopez-Lorenzo ${ }^{2}$, Javier Santos ${ }^{1,2,3, \bullet}$ and \\ Jose Fernandez-Piqueras ${ }^{1,2,3, *,}$,
}

${ }^{1}$ Department of Genome Dynamics and Function, Centro de Biología Molecular Severo Ochoa (CBMSO), Madrid 28049, Spain, ${ }^{2}$ Division of Hematology and Hemotherapy, IIS Fundación Jiménez Díaz, Madrid 28040, Spain, ${ }^{3}$ Consorcio de Investigación Biomédica de Enfermedades Raras (CIBERER), Madrid 28029, Spain, ${ }^{4}$ Division of Cancer, Instituto de Investigación Hospital 12 de Octubre, Madrid 28041, Spain and ${ }^{5}$ Department of Pathology, Hospital Universitario de Canarias, ITB, Universidad de La Laguna, La Cuesta 38320, Spain

\begin{abstract}
*To whom correspondence should be addressed. Centro de Biologia Molecular Severo Ochoa (CBMSO), Consejo Superior de Investigaciones CientíficasUniversidad Autonoma de Madrid (CSIC-UAM), C/ Nicolas Cabrera 1, Campus de la Universidad Autonoma de Madrid, 28049 Madrid, Spain. Tel.: +34 91 196462; Fax: +34 91 1964420; Email: jfpiqueras@cbm.csic.es
\end{abstract}

\begin{abstract}
Precursor T-cell lymphoblastic neoplasms are aggressive malignancies in need for more effective and specific therapeutic treatments. A significant fraction of these neoplasms harbor deletions on the locus 9p21, targeting the tumor suppressor CDKN2A but also deleting the aconitase 1 (ACO1) gene, a neighboring housekeeping gene involved in cytoplasm and mitochondrial metabolism. Here we show that reducing the aconitase activity with fluorocitrate decreases the viability of T-cell lymphoblastic neoplasia cells in correlation to the differential aconitase expression. The consequences of the treatment were evidenced in vitro using T-cell lymphoblastic neoplasia cell lines exhibiting 9p21 deletions and variable levels of ACO1 expression or activity. Similar results were observed in melanoma cell lines, suggesting a true potential for fluorocitrate in different cancer types. Notably, ectopic expression of ACO1 alleviated the susceptibility of cell lines to fluorocitrate and, conversely, knockdown experiments increased susceptibility of resistant cell lines. These findings were confirmed in vivo on athymic nude mice by using tumor xenografts derived from two T-cell lines with different levels of ACO1. Taken together, our results indicate that the non-targeted ACO1 deficiency induced by common deletions exerts a collateral cellular lethality that can be used as a novel therapeutic strategy in the treatment of several types of cancer.
\end{abstract}

\section{Introduction}

T-cell lymphoblastic neoplasms are aggressive hematological malignancies originated from precursor $\mathrm{T}$ lymphoblasts whose differentiation is arrested at different stages of maturation into the thymus. They often manifest with extensive bone marrow and blood involvement (acute T-cell lymphoblastic leukemia, T-ALL), or infrequently with less than $25 \%$ of lymphoblasts in the bone marrow or periphery (T-cell lymphoblastic lymphoma, T-LBL) (1). Genomic and gene expression

Received: May 17 2019; Revised: October 17 2019; Accepted: November 122019

@ The Author(s) 2019. Published by Oxford University Press. All rights reserved. For Permissions, please email: journals.permissions@oup.com. 


\begin{tabular}{ll} 
Abbreviations & \\
\hline qRT-PCR & $\begin{array}{l}\text { quantitative real-time-polymerase } \\
\text { chain reaction }\end{array}$ \\
T-ALL & acute T-cell lymphoblastic leukemia \\
T-LBL & T-cell lymphoblastic lymphoma
\end{tabular}

analyses suggest that T-LBL and T-ALL might be considered as two distinct diseases $(2,3)$. Intensive chemotherapy treatment has gradually improved the outcome of these patients. However, primary resistance or relapse occurs in a significant fraction of patients therefore indicating the need for new treatments $(4,5)$.

Common genetic alterations of T-cell lymphoblastic neoplasms include heterozygous and homozygous 9p21 deletions involving cell cycle regulators p16INK4a and p15INK4B encoded by the CDKN2A/B locus (4), but also many neighboring passenger genes (6). Notably, deletions at the 9p21 region are common events in many other human cancers including glioblastoma (7), breast cancer (8) and melanoma $(9,10)$. Germline mutations in CDKN2A and homozygous deletions at the 9p21 region are particularly common in familial melanoma $(9,11,12)$

It has been established that cancer cells may harbor critical point mutations or deletions in passenger or collateral genes encoding essential functions, which are not primarily involved in causing cancer but are able to generate cellular vulnerability providing new therapeutic strategies (13). Although the potential of synthetic lethality in clinical translation was promising, there are not many examples underlying this strategy. A seminal article by Muller et al. (7) reported the general principles whereby the partial functional redundancy with other paralog genes can be harnessed for detecting cancer vulnerabilities. In glioblastoma cells, they showed that the loss of enolase 1 (ENO1), which encodes the enzyme enolase, makes tumor cells dependent of ENO2 and as such, they were more sensitive than normal cells to the inhibition of ENO2. Two additional works reported that 5-methylthioadenosine phosphorylase/CDKN2A-deleted cancers exhibited enhanced dependency on the protein arginine methyl transferase protein arginine $\mathrm{N}$-methyltransferase 5 and its binding partner WDR77 $(14,15)$. Recently, Dey et al. (16) have provided a new example of tumor-specific lethality in pancreatic cancer, when the common homozygous deletion of the tumor suppressor SMAD4 involves genomic deletion of the mitochondrial malic enzyme 2 (ME2)

Since 9p21 deletion is the most common in T-ALL/LBL, in this work we sought to identify within the region some metabolic-housekeeping gene exhibiting partial redundancy, for which any small-molecule inhibitor was available. In accordance with previous reports, 9p21 deletion encompasses the tumor suppressors CDKN2A and CDKN2B (both at 9p21.3) $(17,18)$ but could also entail passenger genes such as aconitase 1 (ACO1) gene at 9p21.1 (7,19). Interestingly, ACO1 shows a partial functional redundancy with ACO2, another member of this family that is located elsewhere in the mouse and human genomes. Thus, ACO2 might compensate ACO1 deletion. Microarray-based Comparative Genomic Hybridization (aCGH) analysis in a sample series of T-LBL revealed the occurrence of 9p21 deletions involving ACO1 that in turn entailed reduced expression of ACO1. Notably, the treatment with fluorocitrate (an aconitase activity inhibitor) of T-cell lines exhibiting different levels of ACO1 and ACO2 differentially affected their survival and proliferation. These results were confirmed in tumor xenografts and extended in cell lines derived from melanoma, thus highlighting the potential of this strategy in different cancer types.

\section{Materials and methods}

\section{Primary T-LBLs}

Primary T-LBL samples were provided by the Biobanks integrated in the Spanish Hospital Biobanks Network (RetBioH; www.redbiobancos.es) and diagnosed according to the World Health Organization Classification of Hematological Malignancies and the European childhood lymphoma pathology panel recommendations (20). Institutional review board approval was obtained for these studies (references CEI 31-773 and CEI-701260). The participants provided written informed consent in accordance with the Declaration of Helsinki.

\section{Cell lines}

JURKAT (derived from T-ALL) and HEK 293T (derived from kidney) cell lines were purchased from the American Type Culture Collection (Manassas, VA). SUP-T1 (derived from T-LBL), PEER and HPB-ALL (derived from T-ALL) cell lines were obtained from the Leibniz Institute Deutsche Sammlung von Mikroorganismen und Zellkulturen-German Collection of Microorganisms and Cell Cultures (Braunschweig, Germany). SK-MEL-28 and SK-MEL-19 (derived from melanoma) (21) were obtained from Dr. Marisol Soengas (Centro Nacional de Investigaciones Ocológicas). The approximate doubling time of every cell line is $48 \mathrm{~h}$, except for HEK-293T and SK-MEL-19 whose doubling time is $24 \mathrm{~h}$. American Type Culture Collection, Deutsche Sammlung von Mikroorganismen und Zellkulturen and Centro Nacional de Investigaciones Ocológicas routinely perform cell line authentication using short tandem repeat profiling. Cell experimentation was always performed between 3 and 35 passages after thawing and cells were kept in culture not longer than 3 months. Mycoplasma tests were negative both at the beginning and at the end of experimentation.

\section{Quantitative real-time-polymerase chain reaction (qRT-PCR)}

RNA was extracted using the TriPure Reagent (Roche Applied Science, Indianapolis, IN) and quantified with NanoDrop ${ }^{\text {TM }}$ One (Thermo Fisher Scientific, Waltham, MA). The amount of mRNA was determined by quantitative RT-PCR using the High-Capacity RNA-to-cDNA ${ }^{\text {TM }}$ Kit (Applied Biosystems, Foster City, CA). Expression values were determined with Fast SYBR $^{\mathrm{TM}}$ Green Master Mix (Applied Biosystems). Reactions were performed in triplicate with an Applied Biosystems 7300 Real-Time PCR system (Life Technologies, Carlsbad, CA). Expression values of the housekeeping genes (B2M and ACTB) in the same samples were used for normalization using the $2^{-\triangle \Lambda C T}$ method. Oligonucleotides sequences are indicated in Table S1.

\section{Western blot analysis}

Proteins were extracted using Radioimmunoprecipitation assay lysis buffer and quantified with the Bicinchoninic acid Protein Assay (Thermo Fisher Scientific). Spectrophotometric absorbance at $560 \mathrm{~nm}$ was measured using a microplate reader iMARK (Bio-Rad, Hercules, CA). Proteins underwent electrophoresis in sodium dodecyl sulfate-polyacrylamide gel electrophoresis gels and transferred to polyvinylidene fluoride membranes using Trans-Blot $\circledast$ Turbo' Tr Transfer System (Bio-Rad, Hercules, CA). Primary antibodies: ACO1 (developed and provided by Dr. Cuezva, CBMSO, Spain), ACO2 (\#6922), Cleaved Caspase-3 (Asp175) (\#9661), Caspase-3 (\#9662), PARP (\#9542) (Cell Signaling, Danvers, MA), IREB2 (IRP-2 (7H6) sc-33682) (Santa Cruz Biotechnology, Inc., Dallas, TX), ANTI-FLAG (F3165), $\beta$-Actin (A5441) and $\alpha$-Tubulin (T9026) (SigmaAldrich, San Luis, MO). Secondary antibodies: antirabbit and antimouse IgGs (\#7054 and \#7056) (Cell Signaling). Peroxidase activity was analyzed using Western Bright ECL Detection System (Advansta, Menlo Park, CA). ImageQuant LAS 4000 (GE Healthcare Bio-Sciences, Piscataway, NJ) was used for image acquisition and the Scion Image Software (Scion Corporation, NIH, Frederick, MD) for band densitometry.

\section{Aconitase activity assay}

Cytosolic (c-aconitase, ACO1) and mitochondrial (m-aconitase, ACO2) aconitase activities were determined using the Aconitase Activity Assay 
Kit (Sigma-Aldrich). In brief, $20 \times 10^{6}$ cells were centrifuged at $800 \mathrm{~g}$ for $10 \mathrm{~min}$ at $4^{\circ} \mathrm{C}$ to remove insoluble material. The supernatant was used for the c-aconitase assay. For the $\mathrm{m}$-aconitase assay, the supernatant was centrifuged at $20000 \mathrm{~g}$ for $15 \mathrm{~min}$ at $4^{\circ} \mathrm{C}$. Then, the pellet was dissolved in $0.1 \mathrm{ml}$ cold Assay Buffer and sonicated for $20 \mathrm{~s}$. Activity assay was then performed according to the manufacturer's instructions.

\section{Microarray-based aCGH}

aCGH analyses were carried out by NIMGenetics (New Integrated Medical Genetics, Madrid, Spain). Purified DNA from primary samples and cell lines were hybridized against the Human Genome CGH $44 \mathrm{~kb}$ microarrays (Agilent Technologies, Palo Alto, CA) version B, spanning through the entire human genome at a median resolution of around $43 \mathrm{~kb}(24 \mathrm{~kb}$ median probe spacing in Refseq). A commercially available male DNA sample (Promega, Madison, WI) was used as reference. Microarray data analyses were performed with InSilicoArray CGH software-smoothing methods included in gene expression profile analysis suite (http://gepas.bioinfo.cipf. es). We considered heterozygous deletions (including monosomy) when the copy number variation was around -0.5 and homozygous deletions when the copy number variation was -1 .

\section{Ectopic expression of ACO1}

Ectopic expression of ACO1 was performed in SUP-T1 by transfecting these cells with a plasmid vector harboring the cDNA of ACO1 in frame with 3 tandem FLAG epitope tag in the C-terminus. Empty vector (pRP[Exp]-EF1A>3xFLAG/\{Stuffer_300bp\}) and pRP[Exp]EF1A>hACO1[NM_002197.3]/3xFLAG vectors were purchased from VectorBuilder (Chicago, IL). $15 \times 10^{6}$ cells were resuspended in $400 \mu \mathrm{l}$ of Roswell Park Memorial Institute and electroporated with $20 \mu \mathrm{g}$ of plasmid in three pulses of $5 \mathrm{~ms}$ at $270 \mathrm{~V}, 2000 \mu \mathrm{F}$ and $2000 \Omega$ (Gene Pulser MXcell ${ }^{\mathrm{TM}}$ Electroporation System, Bio-Rad).

\section{Knockdown experiments}

Transient ACO1 silencing was performed by transfecting JURKAT cells with MISSION shRNA for ACO1. pLKO.1-Puro non-target shRNA control, pLKO.1-Puro ACO1 shRNA-3 (clone TRCN0000056557) and ACO1 shRNA-4 (clone TRCN0000333172) vectors were purchased from Sigma-Aldrich. $10 \times$ $10^{6}$ cells were resuspended in $500 \mu \mathrm{l}$ of Roswell Park Memorial Institute and electroporated with $40 \mu \mathrm{g}$ of plasmid in two pulses of $10 \mathrm{~ms}$ at 300 $\mathrm{V}, 2000 \mu \mathrm{F}$ and $2000 \Omega$ (Gene Pulser MXcell ${ }^{\mathrm{TM}}$ Electroporation System, Bio-Rad).

\section{Cancer cell xenografts and tumor production}

Four-week-old female athymic Nude-Foxn $1^{\text {nu }}$ mice were purchased from ENVIGO (Cambridgeshire, UK) and maintained under specific pathogenfree conditions in the Animal Facility of the Centre of Molecular Biology Severo Ochoa (CBMSO, Madrid, Spain), according to the European Commission Guidelines (Directive 86/609/CEE) on the use of laboratory animals. After 1-week acclimatization before the experiment, subcutaneous tumor xenografts were established by injection of $5 \times 10^{6}$ SUP-T1 cells stably expressing luciferase into one flank of the animals $(n=6)$. Cells were resuspended in $50 \mu \mathrm{L}$ Roswell Park Memorial Institute and mixed with $50 \mu \mathrm{L}$ Corning Matrigel Matrix (Corning, Bedford, MA) before injection. Tumor volume was evaluated by bioluminescence acquisition of anesthetized mice (isofluorane gas, 1.5\%, Abbott, Madrid, Spain) using an IVIS Lumina II (Caliper Life Sciences, Waltham, MA) after intraperitoneal injection of $150 \mathrm{mg} / \mathrm{kg}$ of body weight of D-luciferin (Promega). The luminescent signal was quantified with the Living Image 3.2 software and expressed as photons/s (average radiance). Animals were scarified for humane reasons when tumors were $15 \mathrm{~mm}$ in diameter (experimental end point).

These procedures have the approval of the Community of Madrid (PROEX 22/15), the Ethic Committee of the Spanish National Research Council (CSIC; 308/2015) and the Ethics Committee of Animal Experimentation (CEEA) of the Centre of Molecular Biology Severo Ochoa (Madrid, Spain) (CEEA-CBMSO-23/191).

\section{Fluorocitrate treatments}

Aconitase inhibition was performed by treatments with fluorocitrate, a specific inhibitor of the Krebs' cycle (22). It has been estimated that the concentration of fluorocitrate needed to inactivate $50 \%$ of the aconitase activity in fresh kidney mitochondria was $24 \mu \mathrm{M}$ and that treatment of sonicated mitochondria with $60 \mu \mathrm{M}$ fluorocitrate is able to reduce aconitase activity by up to $20 \%$ (23).

Fluorocitrate solution was prepared as indicated by Shang et al. (24) in a vehicle solution. Briefly, $240 \mathrm{mg}$ of DL-fluorocitric acid barium salt (Sigma-Aldrich) was dissolved in $30 \mathrm{ml}$ of $0.1 \mathrm{M} \mathrm{HCl} . \mathrm{Ba}^{2+}$ was precipitated by adding 90 drops of $\mathrm{Na}_{2} \mathrm{SO}_{4}(0.1 \mathrm{M})$ and then, $60 \mathrm{ml} \mathrm{Na}_{2} \mathrm{HPO}_{4}(0.1 \mathrm{M})$. The suspension was then centrifuged at $1000 \mathrm{~g}$ for $5 \mathrm{~min}$. The supernatant was diluted with $0.9 \% \mathrm{NaCl}$ to the final concentration of $2 \mathrm{mM}$ and $\mathrm{pH} 6.68$.

Cell lines were treated with fluorocitrate at concentrations ranging from 25 to $500 \mu \mathrm{M}$, using the vehicle as a reference $(0 \mu \mathrm{M})$. Cells were collected at 24,48 and $72 \mathrm{~h}$ after treatment to perform cell viability and proliferation analyses. Cells transfected with a plasmid vector harboring the CDNA of ACO1 or shRNA for ACO1 were treated with fluorocitrate $24 \mathrm{~h}$ after transfection and collected $24 \mathrm{~h}$ after treatment to perform cell viability and proliferation analyses. Xenografts mice were treated with $60 \mathrm{mg} / \mathrm{kg}$ of body weight fluorocitrate, once a day, 5 days a week, starting 1 week after inoculation and until experimental end point. Brain, kidney, liver and spleen biopsies were collected from killed mice and frozen for histological analyses. Samples were fixed with 10\% formalin for $2 \mathrm{~h}$, cryoprotected with $30 \%$ sucrose in phosphate buffer saline for $24 \mathrm{~h}$ and embedded in Tissue-Tek® O.C.T' ${ }^{\text {тM }}$ Compound (Sakura Finetek Europe B.V., Alphen aan den Rijn, The Netherlands). Sections of $3 \mu \mathrm{m}$ were stained with hematoxylin and eosin. Coded slides were studied under the microscope by a pathologist unaware of the codes meaning. Potential pathological changes in the tissues were recorded in a blinded fashion.

\section{Cell death analysis}

Cell death was determined as the number of cells stained with propidium iodine using a FACSCalibur cytometer (Becton Dickinson, San Diego, CA). Data were analyzed by using FlowJo software (TreeStar, Inc., Ashland, OR).

\section{Cell viability and proliferation}

Cell viability was determined using Cell Proliferation Kit I (MTT) (Roche). Spectrophotometric absorbance at $560 \mathrm{~nm}$ with a reference wavelength of $750 \mathrm{~nm}$ was measured using the microplate reader iMARK (Bio-Rad).

\section{Soft agar colony formation assay}

A colony formation assay was performed for semiquantitative evaluation of anchorage-independent growth of melanoma cells lines in response to fluorocitrate treatment. $0.5 \times 10^{4}$ cells were mixed with $0.7 \%$ soft agar in Dulbecco's modified Eagle's medium with increasing fluorocitrate concentrations $(25-500 \mu \mathrm{M})$, poured onto slightly solid $1 \%$ soft agar in medium with fluorocitrate and cultured for a week. Colonies formed in the soft agar were stained with MTT (3-[4,5-dimethylthiazol-2-yl]-2,5-diphenyl tetrazolium bromid) labeling reagent (Roche) for $24 \mathrm{~h}$ at $37^{\circ} \mathrm{C}$ (with $\mathrm{CO}_{2}$ ). Plates were photographed and colonies were quantified using ImageJ Software (NIH, Frederick, MD).

\section{Statistical analyses}

Student's t-test was used to compare results the means from different analyses (qRT-PCR, western blot) from at least three independent experiments. After these analyses, we checked the expression data set for normality distribution using the Shapiro-Wilk test and the homogeneity of variances by Levene's test. Pearson's correlation coefficient and tests were used to determine the statistical correlation between two variables. All statistical analyses were performed using GraphPad Prism 7 (version 7.0; GraphPad Software, San Diego, CA). Tests with P values below 0.05 were considered statistically significant.

\section{Results}

\section{ACO1 is a candidate gene for 9 p21 deletions in T-cell lymphoblastic neoplasia}

On the basis of the information provided by Muller et al. (7) ACO1 was included in a curated list of candidate housekeeping genes with homozygous deletions in glioblastoma cells, redundant 
A

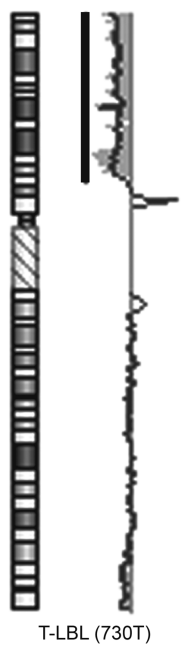

D

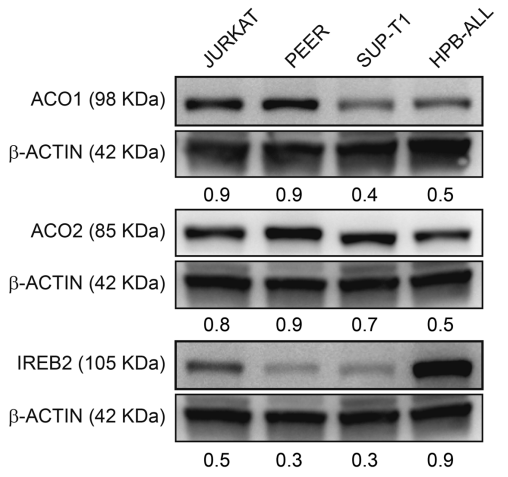

B

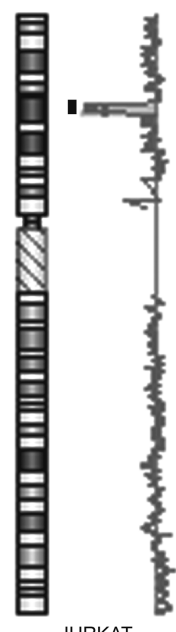

JURKAT

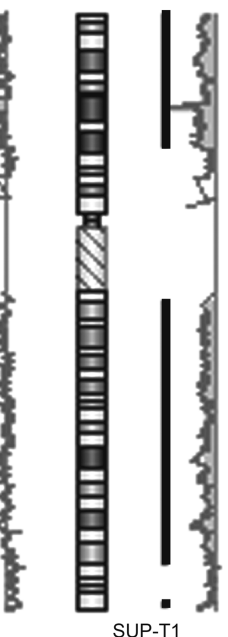

C

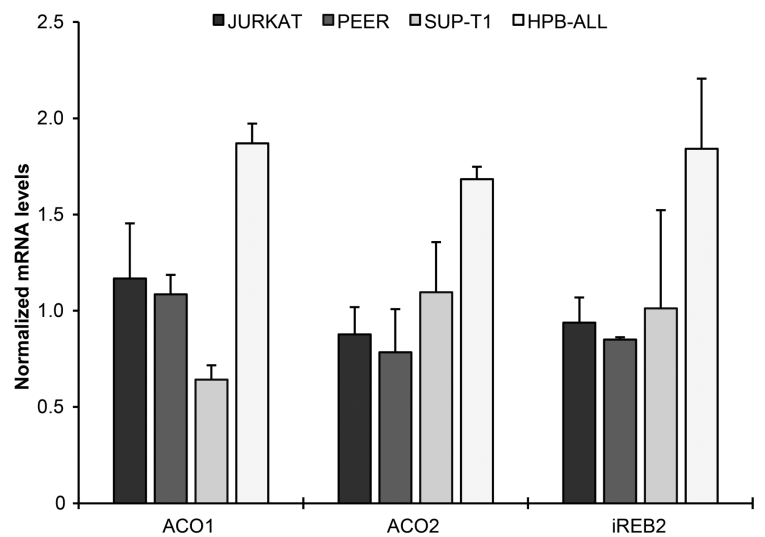

E

Figure 1. Ideograms of microarray-based aCGH analyses (A and B) and evaluation of aconitase expression and activity in cell lines derived from T-cell lymphoblastic neoplasms (C-E). (A) Heterozygous deletion of $66.30 \mathrm{Mb}$ (black bar) in the short arm of chromosome 9 (9p24.2p11.2) represented in a primary T-LBL sample (730T). This deletion affects more than 400 genes including ACO1 (9p21.1). In addition, a homozygous deletion of 0.12 Mb at 9p21.3 involved CDKN2A, CDKN2B and CDKN2B-AS1 genes. (B) Copy number variations at 9p21 in two selected cell lines. JURKAT cells present a homozygous deletion of $2.11 \mathrm{Mb}$ at the short arm (9p21.3) including 21 genes (ACO1 excluded). SUP-T1 cells present a mosaic monosomy of the entire chromosome affecting ACO1. (C) Relative mRNA levels of the three members of the aconitase family determined by qRT-PCR in the T-cell lines. Data represent the mean \pm standard deviation (SD) of three independent experiments normalized with respect to the average value of all cell lines. (D) Representative western blot images of ACO1, ACO2 and IREB2. Numbers below each line result from densitometry analysis. (E) Cytosolic (ACO1) and mitochondrial (ACO2) aconitase activities in cell lines derived from T-cell lymphoblastic neoplasms. One unit indicates the amount of enzyme that will isomerize $1 \mu \mathrm{M}$ of citrate to isocitrate per minute at $\mathrm{pH} 7.4$ at $25^{\circ} \mathrm{C}$. Data represent the mean \pm standard deviation (SD) of three independent experiments. Significant differences were determined by Student's t-test $\left({ }^{*} \mathrm{P} \leq 0.05,{ }^{* *} \mathrm{P} \leq 0.01,{ }^{* * *} \mathrm{P} \leq 0.001\right.$, ns: non-significant).

with paralog genes located elsewhere in the genome and proven targets of small molecules with a potential use in therapy. ACO1 is a housekeeping gene encoding a bifunctional cytosolic protein that catalyzes the conversion of citrate to isocitrate (UniProtKB/ Swiss-Prot ACOC_HUMAN, P21399) and is involved in the 9p21 deletion.

Given that 9p21 deletions are a common feature of T-cell lymphoblastic neoplasia, we first evaluated the involvement of ACO1 copy number variations (deletions) in primary T-LBL samples using microarray-based aCGH. The analysis of a sample series consisting of 19 primary T-LBLs revealed frequent 9p21 causal deletion involving the CDKN2A locus $(8 / 19,42.1 \%)$, and one of them entailing the ACO1 locus at 9p21.1 (Figure 1A). ACO1 paralog genes, ACO2 and IREB2, located elsewhere in the human genome, did not exhibit copy number variations.

In search of an in vitro model, we then investigated a panel of cell lines derived from T-cell lymphoblastic neoplasms. aCGH revealed that SUP-T1 cells experienced a mosaic monosomy affecting the entire chromosome 9. JURKAT cells have a causal homozygous deletion at 9p21.3 that does not involve ACO1 locus (Figure 1B). HPB-ALL cells do not have any disruption involving chromosome 9. None of the cells analyzed exhibited chromosome 22 or chromosome 15 copy number alterations involving ACO2 or IREB2 loci, respectively.

\section{ACO1, ACO2 and IREB2 expression and activity in T-cell lymphoblastic neoplasia cell lines}

The expression levels of ACO1, ACO2 and IREB2 were analyzed in four cell lines derived from T-cell lymphoblastic neoplasms. SUPT1 showed the lowest levels of ACO1 and ACO2 expression (both at mRNA and protein levels). In contrary, JURKAT and PEER cells exhibited the highest levels of ACO1 and ACO2 expression. PEER and SUP-T1 cells expressed the lowest amount of IREB2 protein, which has not aconitase activity. Surprisingly, HPB-ALL cells exhibited low levels of ACO1 protein despite its high level of ACO1 
mRNA, suggesting some unknown post-transcriptional regulatory mechanism (Figure 1C and D). We determined aconitase enzymatic activity in the four cell lines, separating cytosolic aconitase (attributable to ACO1) from mitochondrial aconitase (due to ACO2). Our results indicated that ACO1 activity was lower in SUP-T1 and HPB-ALL cell lines than in JURKAT and PEER (Figure $1 \mathrm{E}$ ), in line with protein levels revealed by western blot.

\section{The aconitase inhibitor fluorocitrate induces a cytotoxic effect of variable degree in T-cell lymphoblastic neoplasia cell lines}

We sought to explore the effect of pharmacological treatments over T-cell lines using fluorocitrate and oxalomalate as two small molecules capable of inhibiting aconitase activity. In the cell, fluorocitrate is converted to fluoro-cis-aconitate and then to 4-hydroxy-trans-aconitate, which binds to protein aconitases to inhibit their enzyme activity (25). On the other hand, oxalomalate is a powerful competitive inhibitor of ACO2 (26) that also cause a decrease in the iron-responsive element of IREB2 (27).

The assessment of propidium iodide positivity by flow cytometry revealed that the treatment with oxalomalate did not produce any significant cell response (data not shown). However, the treatment with fluorocitrate induced a substantial increase in the levels of cell death in SUP-T1 only $24 \mathrm{~h}$ after treatment and with concentrations as low as $25 \mu \mathrm{M}$ (Figure 2A), as well as a significant reduction in the percentage of living cells, as determined using a cell survival/proliferation approach (MTT) (Figure 2B).

The response to fluorocitrate was significantly different among cell lines, as reflected by the percentage of propidium iodine positive cells and MTT values (Figure 2C). Cell lines exhibiting lower ACO1 levels were more sensitive to cell death induction by fluorocitrate (particularly SUP-T1), which manifested with a significantly reduced percentage of living cells after treatment.

Taken together, our results indicate that reduced levels and activity of ACO1 make SUP-T1 cells significantly more vulnerable to fluorocitrate at low concentrations of the drug.

Next, we sought to elucidate whether fluorocitrate-induced cell death could be attributed to apoptosis. For such purpose, two key apoptotic proteins were evaluated by western blot in two cell lines, one susceptible to the treatment (SUP-T1) and the other resistant (JURKAT). Whereas JURKAT cells showed no evidence of apoptosis, proteolytic activation of caspase 3 and poly(ADP-ribose) polymerase-1 (PARP) in SUP-T1 cells was already detectable $24 \mathrm{~h}$ after treatment with $25 \mu \mathrm{M}$ of fluorocitrate. These findings indicate that fluorocitrate-induced cell death in SUP-T1 cells might be, at least in part, attributed to apoptosis (Figure 2D).

\section{The response of tumor cells to fluorocitrate relies on ACO1 levels}

To further confirm whether ACO1 level is the key factor in determining the response of tumor cells to fluorocitrate, we overexpressed ACO1 in SUP-T1 cells and we silenced endogenous ACO1 in JURKAT cells (Figure 3A and B). Overexpression of ACO1 protected SUP-T1 cells from the cytotoxic effect of fluorocitrate (Figure 3C) whereas knockdown of ACO1 increased the sensitivity of JURKAT cells to the drug (Figure 3D). These results support the role of ACO1 level and activity in the vulnerability of T-cell lymphoblastic neoplasia cell lines to fluorocitrate.

\section{In vivo reduction of tumor growth by fluorocitrate}

To study the potential of fluorocitrate as a therapy for cancer patients exhibiting ACO1 deficiency in the tumor cells, we tested the ability of fluorocitrate to reduce the tumor growth in vivo using xenografts. Athymic nude-Foxn $1^{\text {nu }}$ mice bearing SUP-T1-derived tumor xenografts were treated with $60 \mathrm{mg} / \mathrm{kg}$ of fluorocitrate or vehicle 5 days a week for 2 weeks. SUP-T1 cells developed aggressive tumors that did not allow for longer treatments. Fluorocitrate induced a significant reduction of tumor growth at day 15 (Figure 4) in comparison with vehicle. The dosage of fluorocitrate was well tolerated by the animals, which showed normal physical activity and behavior and no symptoms of toxicity. In addition, no pathological changes were detected in tissue sections from brain, liver, kidney and spleen (Supplementary Figure S1, available at Carcinogenesis Online). These results support the use of fluorocitrate as a specific therapy to kill tumor cells in cancer patients with ACO1 deficiency.

\section{ACO1 levels determine the vulnerability of melanoma cells to fluorocitrate}

Since homozygous deletions at the 9p21 region are common in other tumor types, we checked whether low ACO1 levels also induce tumor susceptibility in melanoma. We selected two cell lines derived from melanoma (SK-MEL-28 and SK-MEL-19) exhibiting significant differences in ACO1 copy number. aCGH revealed that SK-MEL-19 cells suffered a partial monosomy affecting the cytobands 9p22.3-9p13.1 that includes the ACO1 gene, whereas SK-MEL-28 does not harbor any deletions affecting chromosome 9 (Figure 5A). Accordingly, SK-MEL-19 showed a significant reduction in ACO1 expression and activity (Figure 5B-D). Using a cell proliferation assay, we evidenced that SK-MEL-19 cell line was significantly more sensitive to fluorocitrate than SK-MEL-28 (Figure 5E). This result was confirmed by anchorage-independent growth assays in response to fluorocitrate, as SK-MEL-19 cell line developed fewer and smaller colonies than SK-MEL-28 cell line (Figure 5F and G and Supplementary Figure S2, available at Carcinogenesis Online). Altogether, these results suggest that reduced ACO1 level and activity induced by copy number reductions at the ACO1 locus might be responsible for the greater susceptibility to the treatment with fluorocitrate also in melanoma.

\section{Discussion}

Two seminal papers outlined the potential of collateral lethality to treat specific types of cancers. The first one experimentally focused on the deletion of the glycolytic gene ENO1 as a passenger gene in the 1p36 deletion, which is compensated by ENO2 expression (7). Five years later the same team returns to the synthetic lethality strategy by reporting a clear example of collateral lethality featured by the genomic deletion of the gene encoding the ME2 in a common deletion involving the tumor suppressor SMAD4 in pancreatic ductal carcinoma (16).

In the present paper, we focused on the ACO1 gene as a candidate passenger gene in the common 9p21 deletion in T-cell lymphoblastic neoplasia. ACO1 is a housekeeping gene that encodes a protein able to catalyze the conversion of citrate to isocitrate in the cytoplasm and to control the levels of iron inside the cells (UniProtKB/Swiss-Prot ACOC_HUMAN, P21399). Two paralog genes (ACO2 and IREB2) show partial redundancy with the ACO1 gene. The ACO2 gene (at 22q13.2) encodes a mitochondrial protein that catalyzes the interconversion of citrate to isocitrate via cis-aconitate in the second step of the Tricarboxylic acid cycle (UniProtKB/Swiss-Prot for ACO2 gene ACON_HUMAN, Q99798). IREB2 (at 15q25.1) encodes an RNAbinding protein without catalytic activity, which binds to ironresponsive elements in ferritin resulting in the repression of 
A

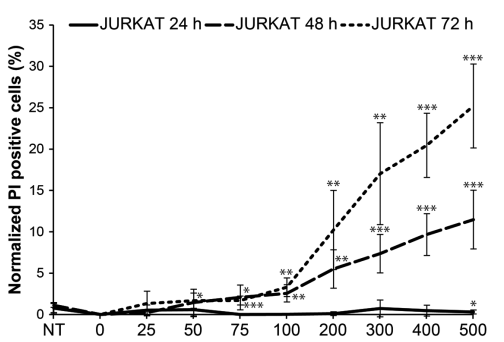

35 - PEER $24 \mathrm{~h}$--PEER $48 \mathrm{~h}$.....PEER $72 \mathrm{~h}$
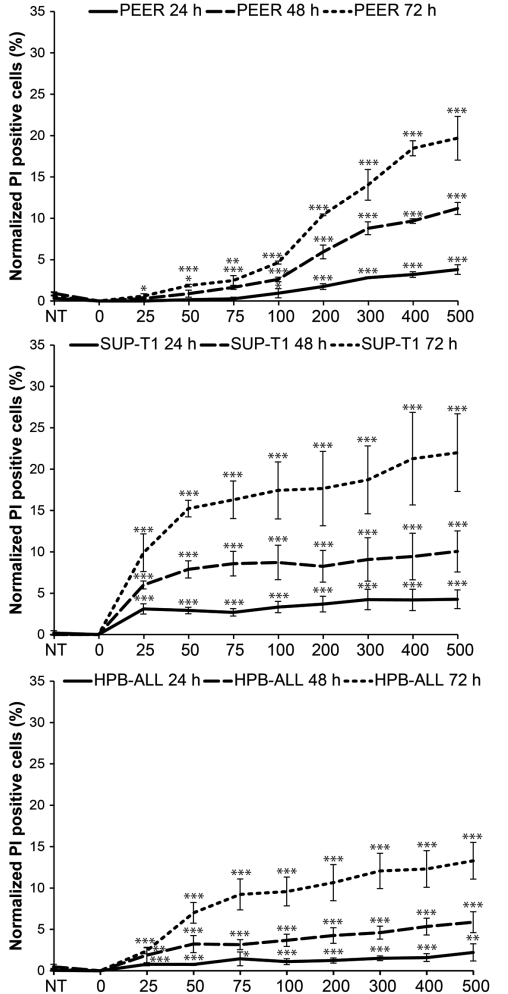

B
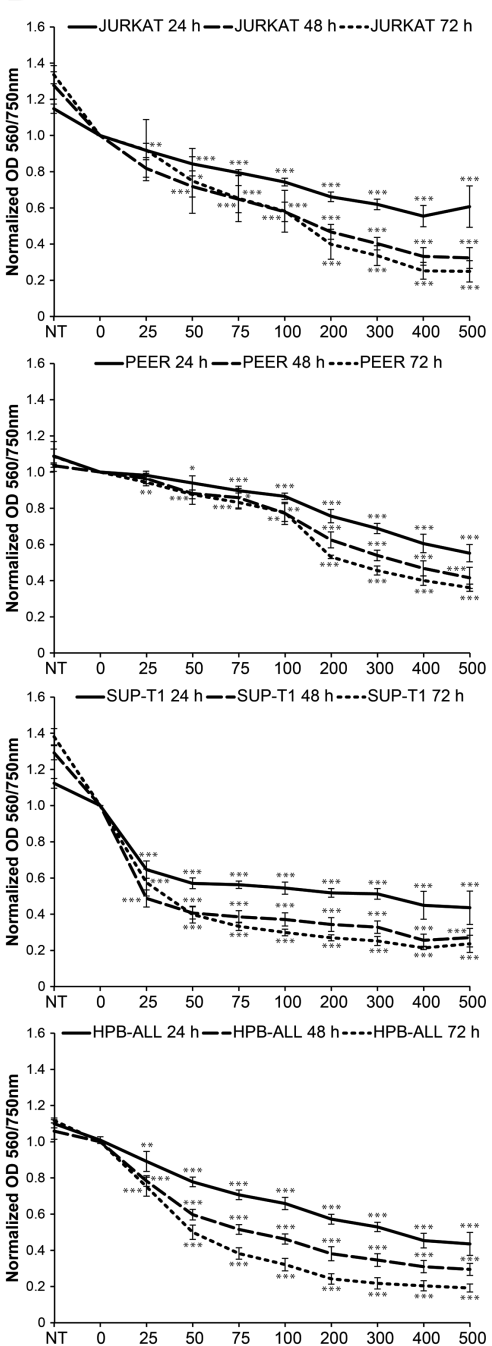

C

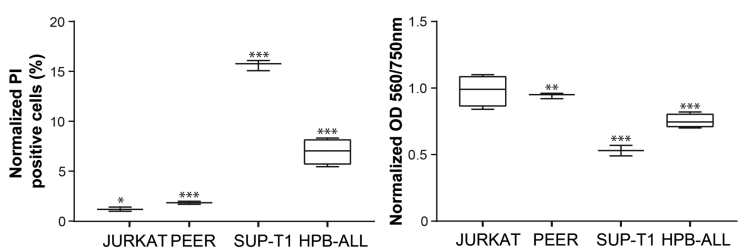

D

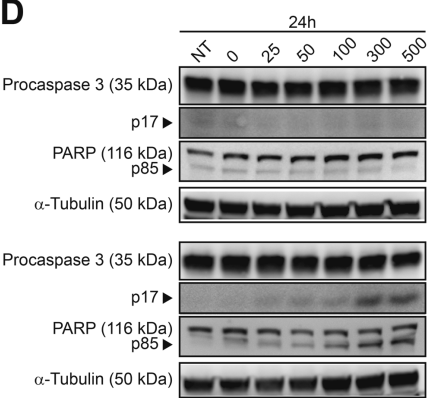

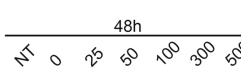

$\frac{72 h}{20 \quad 5_{0} \quad 5^{\circ} \quad 3^{\circ} \quad 5^{\circ}}$
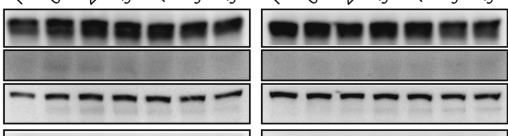

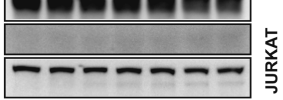

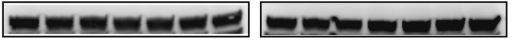
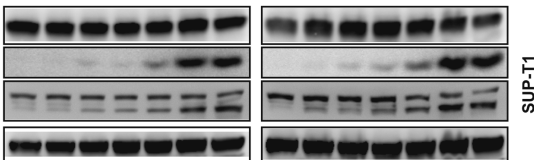

के

Figure 2. Evaluation of the effects caused by fluorocitrate treatment. (A) Flow cytometry analysis of fluorocitrate-treated cells stained with propidium iodide (PI). Cell lines were treated with fluorocitrate $(25-500 \mu \mathrm{M})$ for 24,48 and $72 \mathrm{~h}$. (B) MTT assays following fluorocitrate treatment are represented as the normalized data of the Optical Density (OD) ratio between $560 / 750 \mathrm{~nm}$. (C) Comparison of cell-viability tests among cell lines. Cells treated with $50 \mu \mathrm{M}$ (PI staining, left) or $25 \mu \mathrm{M}$ (MTT assay, right) fluorocitrate were compared at $72 \mathrm{~h}$. (D) Apoptotic induction was evaluated by western blot in cell lines treated with fluorocitrate ( $25-500 \mu \mathrm{M})$. Representative western blots of Caspase 3 activation and PARP proteolysis. NT: non-treated cells. Data represent the mean (A and B) or median (C) \pm standard deviation (SD) of at least three independent experiments normalized to vehicle $(0 \mu \mathrm{M})$. Significant differences were determined using Student's $t$-test $\left({ }^{*} \mathrm{P} \leq 0.05,{ }^{* *} \mathrm{P} \leq 0.01,{ }^{* * *} \mathrm{P} \leq 0.001\right.$, ns: non-significant). 
A

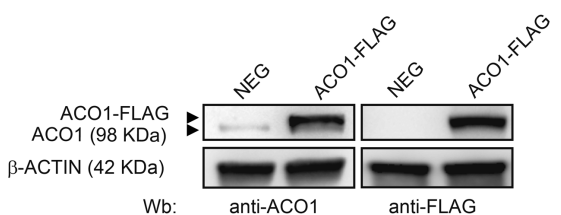

C

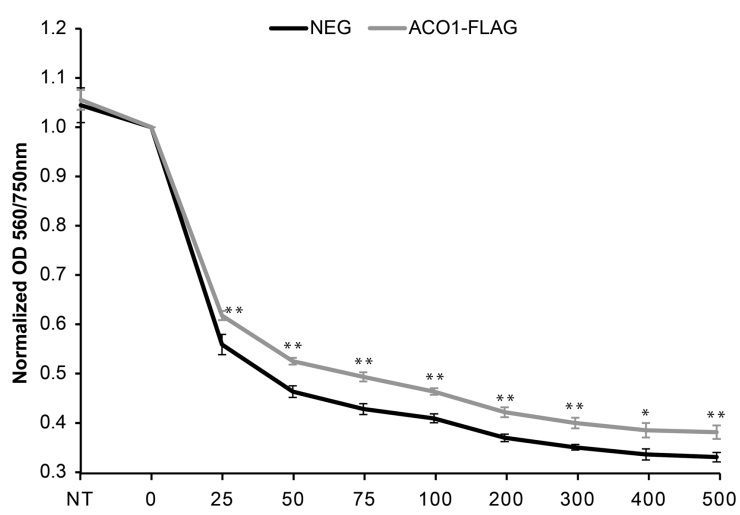

B

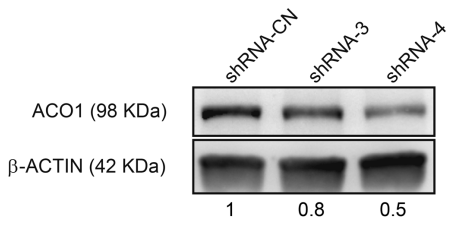

D

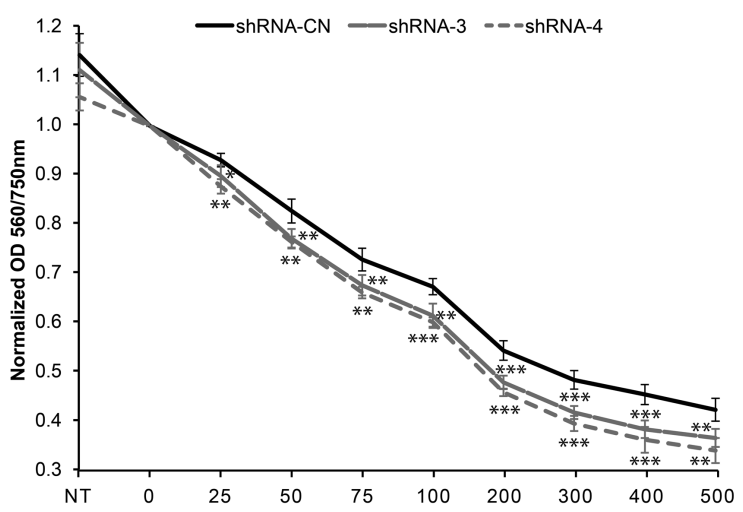

Figure 3. Ectopic expression of ACO1 in SUP-T1 cells and silencing of ACO1 in JURKAT cells. Changes in ACO1 levels were confirmed $48 \mathrm{~h}$ after transfection by western blot with ACO1 and FLAG antibodies (A). NEG, SUP-T1 cells harboring the empty vector; ACO1-FLAG, SUP-T1 cells expressing the same vector harboring ACO1 cDNA. A representative western blot image is shown. (B) shRNA-CN, JURKAT cells harboring the control vector; shRNA-3 and shRNA-4, JURKAT cells expressing the same vector harboring shRNAs against ACO1. A representative western blot image is shown and the numbers depicted below represent the result of densitometry analysis respect to CN. (C and D) MTT assays $24 \mathrm{~h}$ after fluorocitrate treatment (25-500 $\mu \mathrm{M}$ ). Data represent the normalized values of the Optical Density ratio between $560 / 750 \mathrm{~nm}$. NT: non-treated cells. Data represent the mean \pm standard deviation (SD) of at least three independent experiments normalized with respect to vehicle $(0 \mu \mathrm{M})$. Significant differences with respect to NEG (C) and shRNA-CN (D) were determined using Student's t-test $\left({ }^{*} P \leq 0.05,{ }^{* *} P \leq 0.01,{ }^{* * *} P \leq 0.001\right)$.

A

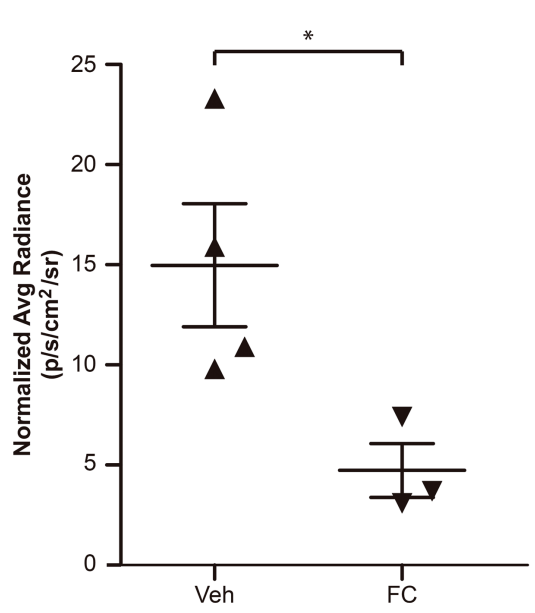

B

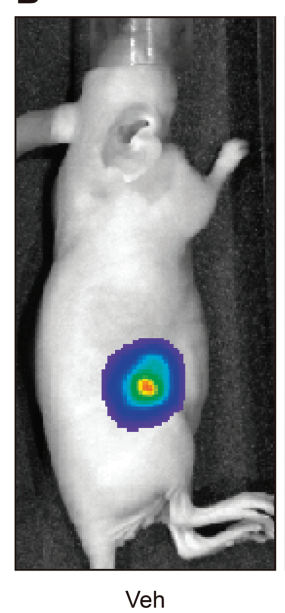

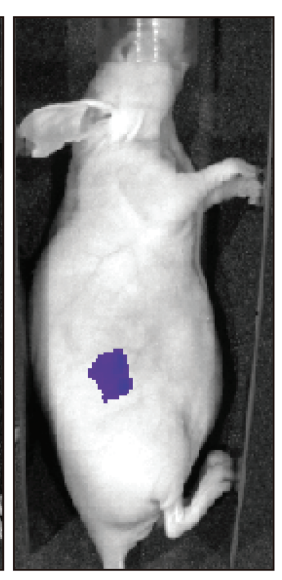

FC

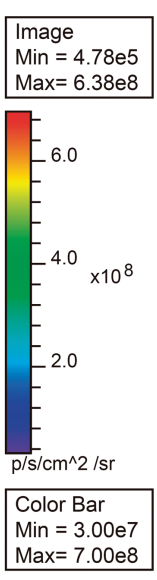

$\operatorname{Max}=7.00 \mathrm{e} 8$

Figure 4. Effect of fluorocitrate in xenograft mice harboring SUP-T1 cells. (A) The growth of SUP-T1 subcutaneous tumors in nude mice was evaluated 15 days after treatment with fluorocitrate by cellular light emission after D-luciferin injection. Data represent the median average radiance \pm standard deviation (SD), normalized to vehicle. Significant differences were determined using Student's t-test $\left(^{*} P \leq 0.05\right)$. (B) Representative images of animals carrying the mentioned tumors, pseudocolored according to bioluminescence intensity. Veh vehicle $(n=4)$; FC, fluorocitrate $(n=3)$.

its mRNA translation. The binding of this protein to the transferrin receptor $\mathrm{mRNA}$ inhibits the degradation of this otherwise rapidly degraded mRNA (UniProtkb/Swiss-Prot for IREB2 gene IREB2_HUMAN, P48200).

Interestingly, multiple knockout investigation in yeast revealed that ACO1 and ACO2 single knockouts are viable but the double knockout condition is lethal (28). In mice, homozygous disruptions of the Aco1 gene display no obvious phenotypic abnormalities (MGI: 87879-aconitase 1) and homozygous disruptions of Ireb2 are viable in spite of some behavioral abnormalities and nervous system degeneration (MGI: 1928268). Combined lethality has been reported for Aco1 and Ireb2 genes (29).

Given these precedents, we focused on deletions at the 9p21 region, a common feature in T-cell lymphoblastic neoplasia. Apparently, the primary oncogenic target of these deletions is the suppressor gene CDKN2A, but they also mobilized other passenger genes within the deleted site such as ACO1. The analysis 
A

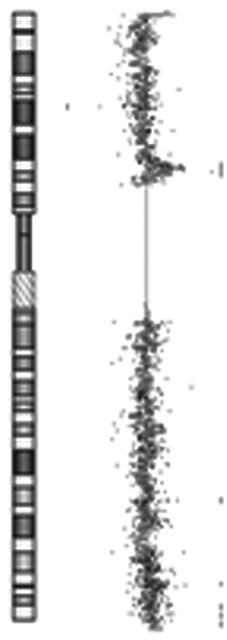

SK-MEL-28

D

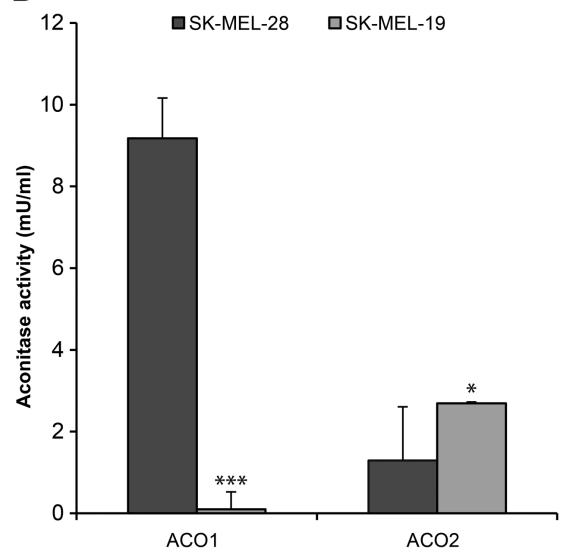

F

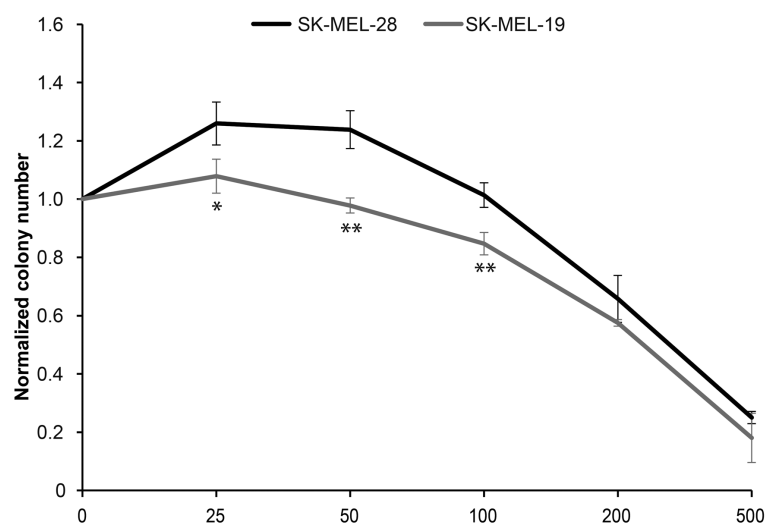

B

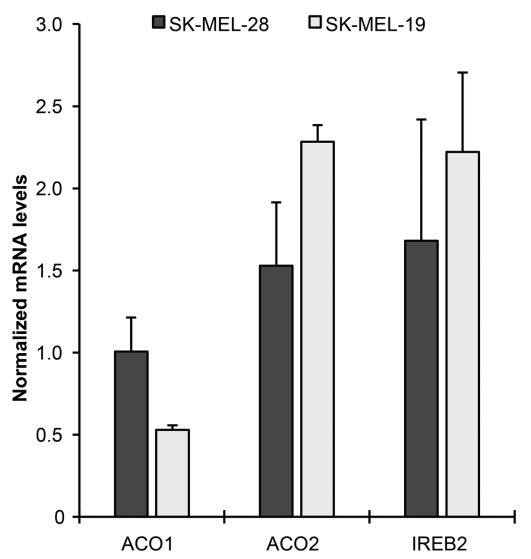

E
C

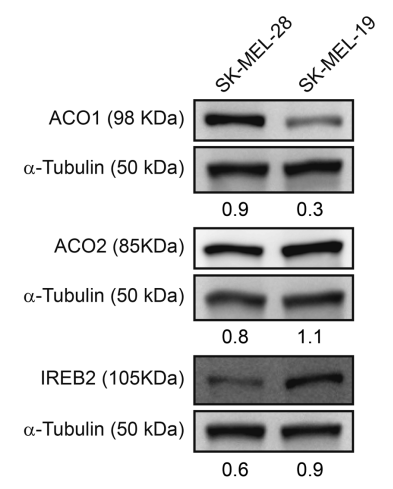

Figure 5. Effects of fluorocitrate treatments in melanoma cells with differences in ACO1 copy number, expression and activity. (A) Ideogram of chromosome 9 resulting from microarray-based aCGH analyses in SK-MEL-28 and SK-MEL-19 cells. Partial monosomy of 23.57 Mb (black bar) at the short arm (p22.3-p13.1) was observed in SK-MEL-19 cells. This modification affects more than 100 genes including ACO1 (9p21.1). (B) Aconitase expression levels of the three members of the family by qRT-PCR. (C) Representative western blots of ACO1, ACO2 and IREB2. Numbers represent the results of densitometry analysis. (D) Cytosolic (ACO1) and mitochondrial (ACO2) aconitase activities. One unit indicates the amount of enzyme that will isomerize $1 \mu \mathrm{M}$ of citrate to isocitrate per minute at pH 7.4 at $25^{\circ} \mathrm{C}$. (E) MTT assays after fluorocitrate treatment $(25-500 \mu \mathrm{M})$. Data represent the normalized data of the Optical Density ratio between 560/750 nm. (F) Soft agar colony formation assay upon fluorocitrate treatment $(25-500 \mu \mathrm{M})$. (G) Representative images of colonies after treatment with fluorocitrate $100 \mu \mathrm{M}$. Veh vehicle; FC, fluorocitrate. Data represent the mean \pm standard deviation (SD) of at least three independent experiments normalized to the average value of SK-MEL-28 and SK-MEL-19 cells (B) or to vehicle (0 $\mu M$ ) (E and F). Significant differences in respect to vehicle $(0 \mu \mathrm{M})(\mathrm{E})$ or to SK-MEL-28 (F) were determined using Student's t-test $\left({ }^{*} \mathrm{P} \leq 0.05,{ }^{* *} \mathrm{P} \leq 0.01,{ }^{* * *} \mathrm{P} \leq 0.001\right)$.

of a panel of cell lines derived from T-cell lymphoblastic neoplasia revealed a complex scenario regarding the expression levels of the three members of the aconitase multigene family
(ACO1, ACO2 and IREB2), although the aconitase activity could be essentially attributed to the ACO1 protein. Thus, a significant decrease in ACO1 expression caused by a deletion in 9p21 would 
generate a vulnerability picture in tumor cells, as they would become more sensitive to the treatment with aconitase inhibitors like fluorocitrate. Moreover, the effect of this drug should be proportional to the aconitase expression and activity in tumor cells.

Among the cell lines analyzed, SUP-T1 exhibits the lowest levels of ACO1 expression and activity and, in addition, it has relatively low levels of IREB2 expression. As hypothesized, these cells were most susceptible to treatment with fluorocitrate, since the aconitase activity was seriously compromised in treated cells. Moreover, it could be speculated that the impaired iron metabolism in these cells would also contribute to their exhaustion upon fluorocitrate treatment.

Given that homozygous 9p21 deletions are common events in multiple hematological and solid malignancies, we extended our analyses to melanoma. In line with T-cell lymphoblastic neoplasia, the reduced levels of ACO1 resulting from 9p21 deletions in SK-MEL-19 also appeared to be a sufficient condition to induce vulnerability to fluorocitrate.

The practical suitability of fluorocitrate as a new treatment to eliminate tumor cells could be handicapped due to its reported high toxicity. However, in vivo experiments in this paper revealed that cells exhibiting ACO1 deficiency (SUP-T1 cells) were highly sensitive to the treatment with low doses of fluorocitrate $(10-100 \mu \mathrm{M})$. Kidney injury is one of the main markers of fluoroacetate ('compound 1080', a fluorocitrate precursor used in pest control) toxicity (30). Extensive kidney studies revealed no signs of tubular coagulative necrosis in our mice.

Our findings extend the proposal by Mavrakis et al. (14) and Kryukov et al. (15) about collateral vulnerability generated by loss of MTAP passenger gene in cancers exhibiting the 9p21 deletion, by adding ACO1 as another passenger gene sustaining this kind of tumor vulnerabilities. Taken together, our results suggest that ACO1 protein deficiency, which results from the 9p21 deletion or another alternative mechanism, generates a vulnerability in tumor cells that can be harnessed to eliminate them by treatment with aconitase inhibitors, while maintaining cell viability of the unaffected surrounding tissues.

An apparent weakness of our proposal is that only a small number of patients with T-cell lymphoblastic neoplasms could be beneficiaries of this treatment. In our limited cohort, 1/19 (5.3\%) patients exhibited ACO1 deletion. However, 9p21 deletions are a fairly frequent event in various hematologic and solid tumors (including melanoma, breast cancer, colorectal carcinoma and so on). We have performed a search by interrogating cBioPortal for tumors with 9p21 deletions involving ACO1. Interestingly, $3.3 \%$ (25/764) of pediatric B-ALL, $0.6 \%$ (5/838) of melanoma patients and $0.3 \%(41 / 13343)$ of patients with breast or lung cancer show deletions that involve ACO1 and, consequently, it could be deduced that they show significant decreases in the expression of ACO1. This indicates that the frequency of ACO1 deletion in T-LBL that we have obtained in our limited cohort is representative of the overall frequency in other cancer types, when analyzed in much larger cohorts as those included in cBioportal. Most interestingly, the number of cancer patients potentially candidate to benefit from the treatment with fluorocitrate rises significantly beyond those with T-LBL.

\section{Supplementary material}

Supplementary data are available at Carcinogenesis online.

\section{Funding}

Instituto de Salud Carlos III (ACCI-CIBERER-17); Spanish Ministerio de Economía y Competitividad (SAF2015-70561-R;
MINECO/FEDER, EU); Spanish Ministerio de Ciencia, Innovación y Universidades (RTI2018-093330-B-I00; MCIU/FEDER, EU); Universidad Autónoma de Madrid, Spain (B2017/BMD-3778; LINFOMAS-CM); Spanish Association Against Cancer (AECC, 2018; PROYE18054PIRI); Fundación Ramón Areces (CIVP19S7917); Institutional grants from Fundación Ramón Areces and Banco de Santander to Centro de Biología Molecular Severo Ochoa are also acknowledged.

\section{Acknowledgements}

We would like to express our gratitude to Maria C. de Arriba and Isabel Sastre for their technical help and to Marisol Soengas (at Spain's National Cancer Research Centre, Centro Nacional de Investigaciones Ocológicas) for providing us with the cells derived from melanoma.

We thank all patients who were willing to donate their samples. Without their support, this research would not be possible. Conflict of Interest Statement: None declared.

\section{References}

1. de Leval, L. et al. (2009) Molecular classification of T-cell lymphomas. Crit. Rev. Oncol. Hematol., 72, 125-143.

2. Burkhardt, B. (2010) Paediatric lymphoblastic T-cell leukaemia and lymphoma: one or two diseases? Br. J. Haematol., 149, 653-668.

3. Mussolin, L. et al. (2014) An aberrant microRNA signature in childhood T-cell lymphoblastic lymphoma affecting CDKN1B expression, NOTCH1 and growth factor signaling pathways. Leukemia, 28, 1909-1912.

4. Belver, L. et al. (2016) The genetics and mechanisms of $\mathrm{T}$ cell acute lymphoblastic leukaemia. Nat. Rev. Cancer, 16, 494-507.

5. Oudot, C. et al. (2008) Prognostic factors for leukemic induction failure in children with acute lymphoblastic leukemia and outcome after salvage therapy: the FRALLE 93 study. J. Clin. Oncol., 26, 1496-1503.

6. Muller, F.L. et al. (2015) Collateral Lethality: a new therapeutic strategy in oncology. Trends Cancer, 1, 161-173.

7. Muller, F.L. et al. (2012) Passenger deletions generate therapeutic vulnerabilities in cancer. Nature, 488, 337-342.

8. Lebok, P. et al. (2016) p16 overexpression and 9p21 deletion are linked to unfavorable tumor phenotype in breast cancer. Oncotarget, 7, 81322-81331.

9. Fountain, J.W. et al. (1992) Homozygous deletions within human chromosome band 9p21 in melanoma. Proc. Natl. Acad. Sci. USA, 89, 10557-10561.

10. Lesueur, F. et al.; French Familial Melanoma Study Group. (2008) The contribution of large genomic deletions at the CDKN2A locus to the burden of familial melanoma. Br. J. Cancer, 99, 364-370.

11. Schadendorf, D. et al. (2015) Melanoma. Nat. Rev. Dis. Primers, 1, 15003.

12. Shain, A.H. et al. (2016) From melanocytes to melanomas. Nat. Reu. Cancer, 16, 345-358.

13. Hartwell, L.H. et al. (1997) Integrating genetic approaches into the discovery of anticancer drugs. Science, 278, 1064-1068.

14. Mavrakis, K.J. et al. (2016) Disordered methionine metabolism in MTAP/ CDKN2A-deleted cancers leads to dependence on PRMT5. Science, 351, 1208-1213.

15. Kryukov, G.V. et al. (2016) MTAP deletion confers enhanced dependency on the PRMT5 arginine methyltransferase in cancer cells. Science, 351, 1214-1218.

16. Dey, P. et al. (2017) Genomic deletion of malic enzyme 2 confers collateral lethality in pancreatic cancer. Nature, 542, 119-123.

17. Hebert, J. et al. (1994) Candidate tumor-suppressor genes MTS1 (p16INK4A) and MTS2 (p15INK4B) display frequent homozygous deletions in primary cells from $\mathrm{T}$ - but not from B-cell lineage acute lymphoblastic leukemias. Blood, 84, 4038-4044.

18. Graux, C. et al. (2006) Cytogenetics and molecular genetics of T-cell acute lymphoblastic leukemia: from thymocyte to lymphoblast. Leukemia, 20, 1496-1510.

19. Schraders, M. et al. (2009) High-resolution genomic profiling of pediatric lymphoblastic lymphomas reveals subtle differences with 
pediatric acute lymphoblastic leukemias in the B-lineage. Cancer Genet. Cytogenet., 191, 27-33.

20. Swerdlow, S.H. et al. (2016) The 2016 revision of the World Health Organization classification of lymphoid neoplasms. Blood, 127, 2375-2390.

21. Gorden, A. et al. (2003) Analysis of BRAF and N-RAS mutations in metastatic melanoma tissues. Cancer Res., 63, 3955-3957.

22. Hassel, B. et al. (1992) Selective inhibition of glial cell metabolism in vivo by fluorocitrate. Brain Res., 576, 120-124.

23. Scandroglio, F. et al. (2014) Metabolic control analysis of mitochondrial aconitase: influence over respiration and mitochondrial superoxide and hydrogen peroxide production. Free Radic. Res., 48, 684-693.

24. Shang, X.L. et al. (2015) Fluorocitrate induced the alterations of memory-related proteins and tau hyperphosphorylation in SD rats. Neurosci. Lett., 584, 230-235.
25. Lauble, H. et al. (1996) The reaction of fluorocitrate with aconitase and the crystal structure of the enzyme-inhibitor complex. Proc. Natl. Acad. Sci. USA, 93, 13699-13703.

26. Adinolfi, A. et al. (1971) Inhibition by oxalomalate of rat liver mitochondrial and extramitochondrial aconitate hydratase. Biochem. J., 125, 557-562.

27. Guo, B. et al. (1994) Iron regulates cytoplasmic levels of a novel ironresponsive element-binding protein without aconitase activity. J. Biol. Chem., 269, 24252-24260.

28. Deutscher, D. et al. (2006) Multiple knockout analysis of genetic robustness in the yeast metabolic network. Nat. Genet., 38, 993-998.

29. Galy, B. et al. (2004) Targeted mutagenesis of the murine IRP1 and IRP2 genes reveals context-dependent RNA processing differences in vivo. RNA, 10, 1019-1025.

30. Chi, C.H. et al. (1996) Clinical presentation and prognostic factors in sodium monofluoroacetate intoxication. J. Toxicol. Clin. Toxicol., 34, 707-712. 